\title{
Schreiben und Zeit / Schreiben und Corona-Zeit
}

blog.kulturwissenschaften.de/schreiben-und-zeit-schreiben-und-corona-zeit/

Britta AckselRicarda Menn

Einige Gedanken und zwei Einladungen

Wie in fast allen Bereichen stellt die Corona-Krise auch für Wissenschaftler*innen in der frühen Berufsphase Probleme und Herausforderungen durch ein Brennglas verstärkt dar: angefangen von dem Bangen um Verlängerungen von Arbeitsverträgen oder Stipendien über Doppelbelastungen von Online-Lehre und Erziehungsaufgaben bis hin zu Isolation durch Mangel von Strukturen im Home-Office. Gleichzeitig setzt aber auch hier eine Selbstoptimierungslogik ein: Jetzt, wo viele berufliche und soziale Verpflichtungen reduziert scheinen, sei ja endlich genug Zeit da, um in aller Ruhe zu schreiben - den lange aufgeschobenen Artikel, das halb fertige Kapitel oder vielleicht auch endlich die Dissertation. Zeit, so wird oft beklagt, fehlt im Alltag von Doktorand*innen: Lehre, Kolloquien, Workshops, Tagungen und Konferenzen, oder nicht zuletzt die Finanzierung des Lebensunterhalts buhlen um die knappe Ressource.

Doch ist es wirklich nur Zeit, die uns - scheinbar - am Schreiben hindert? Und welche Schreibprobleme lassen sich wirklich nur mit einem „Mehr“ an Zeit lösen? Viele Schreibratgeber versprechen, dass man mit kleinen Zeitfenstern seine Qualifikationsschrift in den Griff bekommt: Writing Your Dissertation In Fifteen Minutes a Day von Joan Bolker verankert dieses Angebot programmatisch im Titel, und auch Joli Jensens Write No Matter What schlägt vor, kleinere Zeiteinheiten im Terminkalender zum Schreiben zu blocken, um so Routinen und Gewohnheiten herauszubilden. So verlockend dies klingen mag, eröffnen beide, dass solche kurzen Einheiten langfristig nicht zum Erfolg führen, idealerweise können sie aber den Grundstein für längere Schreibsitzungen legen. Zeit, so lässt sich wenig überraschend resümieren, ist unverzichtbar.

Zeitmanagement ist daher auch eines der Lieblingsgesprächsthemen zwischen Promovierenden unterschiedlicher Disziplinen, dicht gefolgt von weiteren Organisationstipps. Pomodoro-Strategien, Projektmanagement-Tools, Literaturverwaltungsprogramme oder Apps, mit denen man sich selbst die Handynutzung verwehrt, sind nur ein kleiner Ausschnitt. Die Beliebtheit des Themas, so ließe sich vermuten, liegt neben seinem Prokrastinationspotential in seiner Eigenschaft als kleinster gemeinsamer Nenner. Promotionen konzentrieren sich per Definition auf sehr spezifische Themen. Selbst in den Geistes-, Kultur- und Sozialwissenschaften ist bereits stark eingeschränkt, worüber sich etwa eine Anglistin 
und eine Kulturanthropologin promotionsbezogen austauschen können. Doch neben Selbstorganisation gibt es mindestens eine weitere offensichtliche Gemeinsamkeit: das Schreiben.

Obgleich Schreibratgeber boomen- vor allem im englischsprachigen Raum gibt es eine Vielzahl an mehr oder weniger vielversprechenden Ratgebern, die sich zum Beispiel an Stilfragen (Stylish Academic Writing, Helen Sword) oder quantitativen Herausforderungen (How to Write a Lot, Paul J. Silvia) abarbeiten - sind Bestimmungen guten wissenschaftlichen Schreibens ein weniger populäres Gesprächsthema. Dies betrifft zunächst zahlreiche handwerkliche Probleme: Was genau macht „gutes“ Schreiben aus? Ist Schreiben etwas, was man - einer Genielogik folgend - entweder kann oder nicht? Kann man „gutes“ Schreiben überhaupt lernen? Wenn ja, wie und wo? An diese basalen Aspekte schließen sich in einem weiteren Schritt disziplinär spezifische Fragen nach Bewertungsrichtlinien und -Instanzen für gutes Schreiben an.

An deutschen Hochschulen gibt es zahlreiche Beratungsmöglichkeiten für Promovierende, und nicht zuletzt auch für das Schreiben: Die RUB Research School veranstaltet zum Beispiel sowohl in deutscher als auch englischer Sprache verschiedene thematische Schreibworkshops, zumeist ausgelegt für Promovierende verschiedener Fachdisziplinen. Auch das Graduate Center Plus der Universität Duisburg-Essen bot während Corona-Hochzeiten eine Online-Schreibwerkstatt an, welche schnell ausgebucht war. Von der Warteliste aus beobachtet, lag der Fokus nicht auf handwerklichen Fragen des Schreibens, sondern diente vor allem der gemeinsamen Schreibmotivation. Analoge Schreibwochen in unterschiedlichen Ausprägungen sind auch Bestandteil der Programme einiger Begabtenförderwerke. In Jugendherbergen, Bildungszentren oder Tagungshäusern kommen Teilnehmer*innen aus verschiedenen Fachkulturen zusammen, um sich möglichst ungestört und abseits des Alltags auf ihre Promotion zu konzentrieren.

Eine etwas andere Herangehensweise findet man zum Beispiel in Graduiertenkollegs oder strukturierten Promotionsprogrammen - je nach Ausgestaltung und zum Teil in etwas homogeneren disziplinären Zusammenhängen - verankern diese Textdiskussionen und Schreibwerkstätten schon in ihren jeweiligen Programmen. An dieser kurzen Bestandsaufnahme zeigt sich, dass Schreibangebote unterschiedliche Zwecke bedienen können: Vor allem bei größeren Gruppen mit Teilnehmer*innen aus verschiedenen Disziplinen stehen die gemeinsame Zeitorganisation und Schreibmotivation im Vordergrund. Fachspezifische Schreibstrategien und handwerkliche Grundlagen lassen sich hingegen passgenauer in spezifischeren Konstellationen diskutieren.

Mit der Annahme, dass es zumindest für Geistes-, Kultur- und qualitative Sozialwissenschaften eine lohnenswerte Schnittmenge an handwerklichen und strategischen Schreibpraktiken geben kann, haben wir am Kulturwissenschaftlichen Institut im Juni 2020 eine erste digitale Schreibwoche für Promovierende dieser Fachkulturen veranstaltet. Dabei haben wir uns bewusst dagegen entschieden, diese 
Woche nur als digitalen Raum zur Selbstmotivation in Zeiten von Home-Office zu nutzen. Vielmehr war uns wichtig, dass Promovierende - neben der gemeinsamen Zeitstrukturierung - auch die Möglichkeit erhalten, in den Austausch über die Arbeit am eigenen Text zu treten. Während die Vormittage zum eigentlichen Schreiben reserviert waren, wurden die Nachmittage der Schreibwoche in zwei Arbeitsformate geteilt: Dienstag und Donnerstag haben die Teilnehmerinnen (und ja, es haben sich ausschließlich Frauen angemeldet), gemeinsam mit fortgeschrittenen Wissenschaftler*innen aus dem KWI die Themen „Kürzen“ und „TerminologieEntwicklung“ diskutiert. Eingeleitet durch einen Input wurden persönliche Erfahrungen und Strategien ausgetauscht sowie Beispiele besprochen. Montag- und Mittwochnachmittag war den Themen „Forschungsstand und Forschungsfrage“ sowie „Übergänge und Abschlüsse“ gewidmet. Nach einem kurzen Input und gemeinsamen Austausch folgte eine Tandemarbeitsphase. Zwei Promovierende kamen hier jeweils zusammen, um zuvor ausgetauschte und durchgearbeitete Textausschnitte zu besprechen.

Wie aber lässt sich mehr Raum und Zeit für „gutes“ Schreiben und intensivere Textarbeit in den (Schreib-) Alltag integrieren, routinisieren und möglicherweise institutionalisieren? In Gesprächen während und nach der ersten KWI Schreibwoche sind hierzu unterschiedliche Ideen, Ansätze und Beispiele zusammengekommen. Eine naheliegende und von vielen geübte Praxis sind Tandems, die sich, auch außerhalb von Schreibwochen, gegenseitig in der Textarbeit unterstützen. Hierbei hat sich herauskristallisiert, dass eine zu starke fachliche oder thematische Nähe nicht geboten sein muss, da die Partner*innen sonst oft bei rein inhaltlichen Diskussionen verbleiben, die natürlich auch wichtig sind, aber oft dazu führen, dass Sprache und Form zugunsten inhaltlicher Details aus dem Blick geraten. Auch im Studium gibt es Ansätze intensiver Textarbeit. An vielen US-amerikanischen Universitäten werden Studierende nicht nur methodisch und inhaltlich, sondern auch in Bezug auf Stil, Ausdruck und Sprache betreut und Texte etwa erst nach verschiedenen Draftversionen endgültig eingereicht. Potential hat auch das gemeinschaftliche Verfassen von Texten, nicht nur, aber oft insbesondere von Wissenschaftler*innen in verschiedenen Qualifizierungsphasen. Und ja: All dies braucht Zeit, die aus unserer Sicht jedoch gut angelegt ist.

Einladen möchten wir daher zu Diskussion und Austausch über gutes Schreiben und intensive Textarbeit: Wie arbeiten Sie an Texten? Mit welchen Formaten oder Ansätzen haben Sie Erfahrungen gemacht? Thematisieren Sie „gutes Schreiben“ in Lehre und Betreuung von Qualifikationsarbeiten? Wenn ja, wie? Und mit was für Hindernissen haben oder hatten Sie in Ihrer Schreibpraxis zu kämpfen?

Am KWI werden wir zu einigen dieser Fragen zurückkehren und laden Promovierende zu unserer zweiten KWI Schreibwoche vom 12. bis 16. Oktober ein. Alle Informationen finden Sie unter: https://kulturwissenschaften.de/veranstaltung/kwi-schreibwoche-2/ 
Beitrag lizensiert unter einer Creative Commons Namensnennung 4.0 International Lizenz.

SUGGESTED CITATION: Acksel, Britta; Menn, Ricarda: Schreiben und Zeit / Schreiben und Corona-Zeit. Einige Gedanken und zwei Einladungen, in: KWI-BLOG, [https://blog.kulturwissenschaften.de/schreiben-und-zeit-schreiben-und-coronazeit/l, 15.09.2020

DOI: https://doi.org/10.37189/kwi-blog/20200915-0900 


\section{DuEPublico}

Duisburg-Essen Publications online

\section{DEUSISBN R G}

offen im Denken

Ub universitäts bibliothek

Dieser Text wird über DuEPublico, dem Dokumenten- und Publikationsserver der Universität Duisburg-Essen, zur Verfügung gestellt. Die hier veröffentlichte Version der EPublikation kann von einer eventuell ebenfalls veröffentlichten Verlagsversion abweichen.

DOI: $\quad$ 10.37189/kwi-blog/20200915-0900

URN: urn:nbn:de:hbz:464-20200915-094639-0 